\title{
Gender Differences in Intermediate Atherogenic Pathways by Cigarette Smoking among Middle-Aged US Adults
}

\author{
Amy Z. Fan ${ }^{*, 1}$ and James H. Dwyer ${ }^{\S, 2}$ \\ ${ }^{I}$ Division of Adult and Community Health, National Center for Chronic Disease Prevention and Health Promotion, \\ Centers for Disease Control and Prevention, Atlanta, GA 30341, USA \\ ${ }^{2}$ Department of Preventive Medicine, Keck School of Medicine, University of Southern California, Alhambra, \\ California, USA
}

\begin{abstract}
Objective: This study investigates mediating atherogenic pathways caused by cigarette smoking (anthropometric, metabolic, hemodynamic, inflammatory factors) among middle-aged adults and whether there are gender differences in these pathways.

Methods: The data were obtained from the Los Angeles Atherosclerosis Study. The sample consists of 573 middle-aged healthy U.S. adults (age 40-60 yrs). Common carotid arterial intima-medial thickness (IMT) measured by B-mode ultrasound was used as a surrogate indicator for subclinical atherosclerosis.

Results and Conclusion: Besides high levels of low-density lipoprotein cholesterol and total cholesterol, cigarette smoking was also associated with manifestations of metabolic syndrome (central obesity, atherogenic dyslipidemia, sympathetic overactivity, elevated inflammation markers). Most intermediate physiologic profiles for former smokers were similar to those for never smokers, suggesting that smoking effects are partly reversible after quitting. The common atherogenic mediating pathways by smoking for men and women was central obesity. The unique pathway for women was dyslipidemia (low HDL cholesterol and high triglycerides), and the unique pathways for men were elevated levels of LDL cholesterol and total cholesterol, sympathetic overactivity, and elevated inflammation markers.
\end{abstract}

Keywords: Blood lipids, cholesterol, female, inflammation, insulin resistance, LDL, male, smoking.

\section{INTRODUCTION}

The association between cigarette smoking and cardiovascular morbidity and mortality has been well established [1]. The pathophysiology underlying cardiovascular risk caused by smoking is complicated. Smoking is adversely associated with endothelial dysfunction and arterial wall thickening $[2,3]$. Smokingaccelerated atherosclerosis was recognized to be involved in the occurrence and progression of cardiovascular disease [4].

The atherogenic effects by cigarette smoking may be direct [5] or indirect by affecting a set of intermediate variables (i.e., mediators) that contribute to atherosclerosis. Regarding the mediating effects, besides elevated serum total cholesterol and low-density lipoprotein (LDL) cholesterol, cigarette smoking also increase the risk of metabolic syndrome [6,7], which constitutes another important risk factor cluster for atherosclerosis [8].

The reports on the association between cigarette smoking and some manifestations of metabolic syndrome are inconsistent [9-13]. Furthermore, the relative difference on

\footnotetext{
*Address correspondence to this author at the Division of Adult and Community Health, National Center for Chronic Disease Prevention and Health Promotion, Centers for Disease Control and Prevention 4770 Buford Hwy NE, MS k66, Atlanta, GA 30341, USA; Tel: (770) 488-5327;

Fax: (770) 488-8150; E-mail: afan@prev.org

${ }^{\S}$ Dr. Dwyer is deceased.
}

1876-5068/09 atherosclerosis and relevant risk factor profiles between current, former, and never smokers are not fully clear [14-16]. More evidence on the extent and on what aspects smoking cessation is related to reduced (or elevated) atherosclerotic risk is needed. In addition, it is controversial whether smoking causes atherosclerosis for men to a greater degree than for women [17, 18]. There are reports that some harmful effects of smoking on the arterial wall and atherogenic intermediate variables are gender-related [19-22]. However, the evaluation of the gender differences in the smoking-atherosclerosis association has rarely included the atherosclerosis markers and mediating variables simultaneously.

In the current study, common carotid arterial intima-medial thickness (CCA-IMT) measured by B-mode ultrasound was used as a surrogate indicator of subclinical atherosclerosis [23]. Increased carotid IMT has been shown to be directly associated with an increased risk of cardiovascular disease [24-26].

This study aimed to investigate: 1) mediating atherogenic pathways caused by cigarette smoking (anthropometric, metabolic, hemodynamic, inflammatory markers) among middle-aged adults, 2) whether adverse effects of smoking are reversible following smoking cessation, and 3) whether there are gender differences in these pathways.

\section{METHODS}

\section{Study Cohort and Follow-Up}

The initial Los Angeles Atherosclerosis Study (LAAS) cohort of 573 middle-aged adults (men, $n=304$, age 40-60 yr; 
Table 1. Baseline Demographic and Physiologic Characteristics of Study Participants by Gender. Los Angeles Atherosclerosis Study, 1995-1998

\begin{tabular}{|c|c|c|c|}
\hline Variables & $\operatorname{Men}(n=304)$ & Women $(n=269)$ & $p^{*}$ \\
\hline & \multicolumn{2}{|c|}{ No. (\%) of Participants } & \\
\hline Ethnicity & & & .12 \\
\hline Hispanic & $100(32.9)$ & $72(26.8)$ & \\
\hline Non-Hispanic white & $166(54.6)$ & $149(55.4)$ & \\
\hline Black & $13(4.3)$ & $18(6.7)$ & \\
\hline Asian & $15(4.9)$ & $27(10.0)$ & \\
\hline Others & $10(3.3)$ & $3(1.1)$ & \\
\hline Smoking status & & & .005 \\
\hline Current smokers & $90(29.6)$ & $53(19.7)$ & \\
\hline Former smokers & $90(29.6)$ & $69(25.7)$ & \\
\hline Never smokers & $124(40.8)$ & $147(54.7)$ & \\
\hline Diabetes & $9(3.0)$ & $7(2.6)$ & .78 \\
\hline Antihypertensive medication & $42(13.8)$ & $51(19.0)$ & .12 \\
\hline Lipid-lowering medication & $27(8.9)$ & $10(3.7)$ & .01 \\
\hline Menopause & - & $142(61.0)$ & - \\
\hline Use of oral contraceptives & & & - \\
\hline Current users & - & $7(3.0)$ & \\
\hline Former users & - & $173(74.6)$ & \\
\hline Use of HRT & & & - \\
\hline Current users & - & $110(47.4)$ & \\
\hline Former users & - & $22(9.5)$ & \\
\hline \multirow[t]{2}{*}{ History of hysterectomy } & - & $82(35.3)$ & - \\
\hline & \multicolumn{2}{|c|}{ Mean (SD) } & \\
\hline Age, y & $48.7(4.7)$ & $51.4(4.4)$ & $<.0001$ \\
\hline Body height, m & $1.76(.07)$ & $1.62(.07)$ & $<.0001$ \\
\hline Mean IMT, $\mu \mathrm{m}$ & $676(105)$ & $654(88)$ & $<.0001$ \\
\hline Fasting insulin, $\log (\mathrm{pmol} / \mathrm{L}) \dagger$ & $4.69(0.57)$ & $4.58(0.47)$ & .0057 \\
\hline Fasting glucose, $\log (\mathrm{mmol} / \mathrm{L}) \dagger$ & $1.74(0.19)$ & $1.68(0.14)$ & .0001 \\
\hline Body mass index, $\mathrm{kg} / \mathrm{m}^{2}$ & $28.5(4.8)$ & $27.2(5.9)$ & .002 \\
\hline Sagittal-transverse abdominal diameter ratio & $0.681(0.055)$ & $0.642(0.054)$ & $<.0001$ \\
\hline Fasting HDL-C, mmol/L & $1.31(0.24)$ & $1.66(0.37)$ & $<.0001$ \\
\hline Triglycerides, $\log (\mathrm{mmol} / \mathrm{L}) \dagger$ & $0.59(0.55)$ & $0.35(0.56)$ & $<.0001$ \\
\hline Fasting LDL-C, mmol/L & $3.68(0.90)$ & $3.24(0.87)$ & $<.0001$ \\
\hline Fasting total serum cholesterol, $\mathrm{mmol} / \mathrm{L}$ & $5.65(0.99)$ & $5.50(0.93)$ & .031 \\
\hline Systolic blood pressure, mmhg & $129.6(12.7)$ & $127.7(16.3)$ & .004 \\
\hline Diastolic blood pressure, mmhg & $91.8(8.9)$ & $88.4(9.8)$ & $<.0001$ \\
\hline C-reactive protein, $\log (\mathrm{mg} / \mathrm{dL}) \dagger$ & $0.294(0.063)$ & $0.680(0.068)$ & $<.0001$ \\
\hline
\end{tabular}

Note. IMT indicates intima-medial thickness; HDL-C, high-density lipoprotein cholesterol; LDL-C, low-density lipoprotein cholesterol. *Gender differences in continuous and categorical variables were determined by analysis of covariance (adjusted for age) and $X^{2}$ test of significance, respectively. $\dagger$ A logarithmically transformed mean and its standard deviation are shown.

women, $\mathrm{n}=269$, age $45-60 \mathrm{yr}$ ) was recruited from random samples of a local utility company employees [27-29]. Hispanics and current smokers were oversampled. The participation rate was $85 \%$. The exclusion criteria were: history of heart attack, angina, revascularization, stroke, or current cancer treatment. The baseline examination was completed in 1995. Two follow-up examinations were completed at 1.5-yr intervals. Out of the selected sample, 500 completed the 18 -month $(n=480), 36$-month $(n=447)$, or both follow-up examinations. The IMT progression was determined by the difference between baseline and 36-month (or 18-month if 36-month not available) follow-up after adjustment for the time between the two examinations. Fully informed consent was obtained from all the participants. The study protocol was approved by the Institutional Review Board of the Keck School of Medicine at the University of Southern California. The baseline characteristics of the study participants are listed in Table 1. Approximately 47\% of the participants were never smokers, with approximately equal proportions of current and former smokers $(26 \%)$. 


\section{Predictor}

Smoking status was defined as former, current, and never smokers. Former smokers were smokers who had already quit smoking before enrollment and remained abstinent during the follow-up.

\section{Measurement of Potential Mediators}

Fasting blood samples were collected by venipuncture and were frozen at $-70^{\circ} \mathrm{C}$. Total serum cholesterol, highdensity lipoprotein (HDL) cholesterol: triglycerides were measured by an autoanalyzer with the Roche direct HDLcholesterol method which meets the 1998 NIH/NCEP goals for acceptable performance [30]. Low-density lipoprotein cholesterol (LDL-C) was estimated for fasting samples only (fasting time longer than 8 hours prior to examination) [31]. Plasma glucose concentration was measured by the glucose oxidase method with a Beckman glucose analyzer (Beckman Instruments, Fullerton, CA). Plasma insulin was determined by a specific radioimmunoassay with reagents from Linco Research (St. Louis, MO) with a detection limit of 2 $\mathrm{microU} / \mathrm{ml}(12 \mathrm{pmol} / \mathrm{l})$ and interassay coefficient of variation of $6-8 \%$. High sensitivity C-reactive protein (Hs-CRP) was measured by Latex particle enhanced immunoturbidimetric assay (ITA) using an automated chemistry analyzer (Equal Diagnostics Company, Exton, PA).

Systolic blood pressure (SBP) and diastolic blood pressure (DBP) were measured by standard sphygmomanometer and recorded to the nearest digit. Pulse pressure (PP) was the difference of SBP and DBP. Seated heart rate was assessed by measuring pulse at seated position. Two measurements were taken for SBP, DBP and heart rate and the average was used for each examination. Height, weight, sagittal abdominal diameter, and transverse abdominal diameter were measured. BMI was calculated as weight $(\mathrm{kg})$ divided by height squared $\left(\mathrm{m}^{2}\right)$. The sagittaltransverse abdominal diameter ratio was obtained from the two diameters.

\section{Outcome Measures}

Common carotid artery intima-medial thickness (CCAIMT) was assessed using high-resolution B-mode ultrasound (ATL scanner, model UM4+, with $7.5 \mathrm{MHz}$ linear transducer). IMT was measured at the distal wall of the artery in the $1 \mathrm{~cm}$ section of the common carotid 0.25 proximal to the bulb. The subjects were scanned in two body positions (supine and lateral) and on both sides (right and left). Up to 8 frames were processed for each subject. Procedures for image acquisition and processing were reported previously [32]. The annual progression rate was calculated from the difference between baseline and 3-yr (or 1.5-yr if 3-yr measurement was missing) follow-up thickness reading after adjustment for the time between the two examinations.

\section{Statistical Analysis}

When considering the mediated (indirect) effects of cigarette smoking, we presume that some mediating variables (anthropometric, metabolic, hemodynamic, inflammatory markers) are more proximate in the atherogenic pathways than cigarette smoking. The effect of cigarette smoking (a distant variable) is passed on to atherosclerosis through those mediating variables, i.e., smoking->mediators->IMT. According to conventional definition, three criteria must be met to determine mediation [33]: (a) there must be a significant relation between smoking status and IMT (or IMT progression), (b) there must be a significant relation between smoking status and the mediating variable, (c) the mediator must be a significant predictor of IMT (or IMT progression) in an equation including both the mediator and smoking status.

Logarithmic transformation was performed on variables (fasting insulin, glucose, triglycerides, C-reactive protein) that were markedly skewed toward high values.

Least-square means (adjusted means) for IMT, IMT progression rate and potential mediators were obtained relative to smoking status groups after adjustment for age, ethnicity, diabetes status, use of antihypertensive and cholesterol-lowering medications. This analysis was carried out to examine the first two criteria of mediation, i.e., a significant relation between smoking status and IMT (and IMT progression) and a significant relation between smoking status and mediating variables. A separate set of analysis was performed for women with further adjustment for menopausal status, oral contraceptive use, and use of hormone replacement therapy.

Partial correlation analysis was performed to further examine the third criteria of mediation, i.e., whether a potential mediator is correlated to IMT and IMT progression after controlling for smoking status.

All analyses were performed in SAS 8.02 (SAS Institute Inc., Cary, NC). All reported $p$ values are two-tailed with significance defined as $p<0.05$. Experimentwise error rate was set at .05 for Bonferroni correction when multiple groups were compared.

\section{RESULTS}

Current smoking was significantly related to greater carotid average IMT for men and more rapid IMT progression for women, compared with never smokers. For both men and women, current smoking was significantly associated with higher LDL cholesterol, total cholesterol, and central obesity (indicated by higher sagittal-transverse abdominal diameter ratio). Current smoking was significantly associated with elevated seated heart rate and C-reactive protein for men. In comparison, current smoking was significantly associated with higher triglycerides and lower HDL cholesterol for women (Table 2).

Most cardiovascular risk factor levels were comparable between former smokers and never smokers. However, the carotid average IMT for former smokers was significantly greater than that for never smokers among men; former smoking was significantly associated with general obesity (indicated by BMI, compared with current and never smokers), central obesity (compared with never smokers); Cigarette smoking (current or former) was not significantly related to fasting plasma levels of insulin, glucose, or any blood pressure measures for both men and women.

The proposed potential mediators were all significantly correlated to either IMT or IMT progression in men and/or women after controlling for smoking status and other covariates (Table $\mathbf{3}$ ). 
Table 2. Summary of Potential Mediators and Outcomes by Smoking Status in Men and Women. Los Angeles Atherosclerosis Study, 1995-1998

\begin{tabular}{|c|c|c|c|}
\hline & Never Smokers & Former Smokers & Current Smokers \\
\hline Men $(n=304)$ & $\mathrm{n}=124$ & $\underline{\mathrm{n}=90}$ & $\underline{\mathrm{n}=90}$ \\
\hline IMT progression, $\mu \mathrm{m} / \mathrm{yr}$ & $9.0 \pm 1.8$ & $9.9 \pm 2.1$ & $9.6 \pm 2.2$ \\
\hline Fasting insulin, $\log (\mathrm{pmol} / \mathrm{L})^{*}$ & $4.651 \pm 0.052$ & $4.785 \pm 0.059$ & $4.641 \pm 0.062$ \\
\hline Seated heart rate, beats/min & $76.3 \pm 1.1^{\mathrm{c}}$ & $77.2 \pm 1.2^{b}$ & $82.6 \pm 1.3^{\mathrm{bc}}$ \\
\hline Hs-CRP, $\log (\mathrm{mg} / \mathrm{dL})^{*}$ & $0.010 \pm 0.095^{\mathrm{c}}$ & $0.196 \pm 0.107^{b}$ & $0.621 \pm 0.112^{\mathrm{bc}}$ \\
\hline Women $(\mathrm{n}=269)$ & $\mathrm{n}=147$ & $\underline{\mathrm{n}=69}$ & $\underline{\mathrm{n}=53}$ \\
\hline Carotid average IMT, $\mu \mathrm{m}$ & $645.9 \pm 7.0$ & $658.3 \pm 10.2$ & $670.8 \pm 12.0$ \\
\hline HDL-C, mmol/L & $1.693 \pm 0.030^{c}$ & $1.705 \pm 0.044^{b}$ & $1.483 \pm 0.054^{\mathrm{bc}}$ \\
\hline $\mathrm{TG}, \log (\mathrm{mmol} / \mathrm{L})^{*}$ & $0.277 \pm 0.043^{\mathrm{c}}$ & $0.304 \pm 0.063^{b}$ & $0.603 \pm 0.076^{b c}$ \\
\hline Seated heart rate, beats/min & $79.7 \pm 0.9$ & $78.7 \pm 1.4$ & $79.9 \pm 1.6$ \\
\hline Hs-CRP, $\log (\mathrm{mg} / \mathrm{dL})^{*}$ & $0.645 \pm 0.088$ & $0.830 \pm 0.128$ & $0.863 \pm 0.153$ \\
\hline Men and Women $(\mathrm{n}=573)$ & $\underline{\mathrm{n}=271}$ & $\underline{\mathrm{n}=159}$ & $\underline{\mathrm{n}=143}$ \\
\hline Fasting glucose, $\log (\mathrm{mmol} / \mathrm{L})^{*}$ & $1.705 \pm 0.009$ & $1.711 \pm 0.012$ & $1.705 \pm 0.013$ \\
\hline BMI, $\mathrm{kg} / \mathrm{m}^{2}$ & $27.58 \pm 0.31^{\mathrm{a}}$ & $28.75 \pm 0.40^{\mathrm{ab}}$ & $27.26 \pm 0.44^{b}$ \\
\hline Rsag2Tr & $0.653 \pm 0.003^{\mathrm{ac}}$ & $0.666 \pm 0.004^{a}$ & $0.672 \pm 0.005^{c}$ \\
\hline LDL-C, $\mathrm{mmol} / \mathrm{L}$ & $3.428 \pm 0.056^{\mathrm{c}}$ & $3.330 \pm 0.072^{b}$ & $3.688 \pm 0.083^{\mathrm{bc}}$ \\
\hline
\end{tabular}

Note. Values are given as least-square mean \pm standard error. The least-square means (adjusted means) were computed using GLM in SAS with adjustment for age, ethnicity, diabetic status, and use of antihypertensive and cholesterol-lowering medications. The analysis was performed for women and men separately if the smoking by sex interaction was detected. The variables with the same superscriptions across groups are significantly different with experimentwise error rate at .05 according to modified Bonferroni method.

Rate of change in IMT was determined by three examinations. All other variables were taken from baseline examination. IMT, intima-medial thickness; BMI, body mass index; Rsag2Tr, sagittal/ transverse abdominal diameter ratio; HDL-C, high-density lipoprotein cholesterol; TG, triglycerides; LDL-C, low-density lipoprotein cholesterol; SBP, systolic blood pressure; DBP, diastolic blood pressure; Hs-CRP, high-sensitivity C-reactive protein. * A logarithmically transformed mean and its standard error are shown.

Based on the three criteria of mediation, the common atherogenic mediating pathways by smoking for men and women was central obesity; The unique pathway for women was dyslipidemia (low HDL cholesterol and high triglycerides), and the unique pathways for men were elevated levels of LDL cholesterol, total cholesterol, sympathetic overactivity (indicated by elevated seated heart rate), and inflammation (indicated by $\mathrm{C}$-reactive protein).

For women, a separate analysis was performed with further adjustment for menopausal status, oral contraceptive use, and use of hormone replacement therapy. No dramatic differences were observed in comparison with the aforementioned results.

\section{DISCUSSION}

The main finding of this study was that smoking was associated with a series of risk factors that mediated early atherosclerosis. Some of the mediating pathways were common across gender, and others were gender-specific.

The lack of association between habitual cigarette smoking and fasting insulin may not necessarily indicate that smoking is not related to insulin resistance [11]. First, hyperinsulinemia may not be an ideal marker for insulin resistance [34]. Second, the epidemiological evidence linking smoking with insulin resistance is considerable. Studies using more accurate measures on insulin resistance (e.g., euglycemic clamp technique, frequently sampled intravenous glucose tolerance test) concluded that smoking is indeed related to insulin resistance [7,35]. Third, smoking may interfere with beta-cell function. Cigarette smoking men had a lower homeostasis model assessment (HOMA) value than ex-smokers and never smokers [36]. There was evidence that current smoking was associated with lower fasting insulin compared with that in nonsmokers [13]. The 
present study also indicated that current smoking was associated with lower fasting insulin compared with that in former smokers. Thus, the simultaneous effects of damaged insulin secretion and increased insulin resistance caused by smoking may diminish the differences of fasting insulin levels between smokers and non-smokers and may even reverse the relations. This may be the underlying cause of the dissociation between smoking and hyperinsulinemia [13].

Table 3. Partial Correlation Coefficients Between IMT, IMT Progression and Potential Mediators. Los Angeles Atherosclerosis Study, 1995-1998

\begin{tabular}{|l|c|l|l|c|}
\hline \multirow{2}{*}{} & \multicolumn{2}{|c|}{ IMT } & \multicolumn{2}{c|}{ IMT Progression } \\
\cline { 2 - 5 } & Women & Men & Women & Men \\
\hline \hline Fasting insulin & $0.228 * * *$ & $0.189 * * *$ & 0.049 & 0.020 \\
Fasting glucose & $0.131+$ & 0.030 & -0.069 & -0.007 \\
BMI & $0.409 * * *$ & $0.202 * *$ & 0.073 & $0.127 *$ \\
RSag2Tr & $0.288 * * *$ & $0.158 *$ & 0.003 & -0.053 \\
HDL-C & $-0.215 * *$ & -0.093 & 0.013 & $-0.141 *$ \\
TG & $0.130+$ & $0.128 *$ & $0.133+$ & 0.032 \\
LDL-C & -0.024 & $0.216 * *$ & 0.035 & 0.042 \\
Total cholesterol & -0.056 & $0.160 *$ & 0.088 & 0.064 \\
SBP & $0.338 * *$ & $0.294 * * *$ & 0.100 & 0.055 \\
DBP & 0.076 & $0.311 * * *$ & -0.068 & 0.092 \\
Pulse pressure & $0.388 * * *$ & $0.107+$ & $0.194 * *$ & -0.013 \\
Seated heart rate & -0.042 & 0.061 & -0.078 & $0.144 *$ \\
Hs-CRP & $0.176 * *$ & $0.141 *$ & $0.129+$ & -0.096 \\
\hline
\end{tabular}

Note. The Pearson partial correlation coefficients were obtained with adjustment for smoking status, age, body height, ethnicity, diabetic status, and use of antihypertensive and cholesterol-lowering medications. $+p<0.10 * p<0.05 * * p<0.01 * * * p<0.001$. IMT, intima-medial thickness; BMI, body mass index; Rsag2Tr, sagittal/ transverse abdominal diameter ratio; HDL-C, high-density lipoprotein cholesterol; TG, triglycerides; LDL-C, low-density lipoprotein cholesterol; SBP, systolic blood pressure; DBP, diastolic blood pressure; Hs-CRP, high-sensitivity C-reactive protein.

Elevated sympathetic nervous system activity is a characteristic trait of the metabolic syndrome $[37,38]$ and a risk factor for atherosclerosis [39]. An acute effect of cigarette smoking is to increase the activity of the sympathetic nervous system and the levels of circulating catecholamines [40, 41]. In habitual smokers, smoking one cigarette increases heart rate, blood pressure and cardiac output [42]. The current study showed no evidence of habitual smoking as a risk factor for hypertension, which is consistent with some of other studies [22]. However, current smoking was indeed associated with elevated habitual heart rate (for men only), which is in agreement with other studies [42].

The gender differences on the atherogenic pathways caused by cigarette smoking have important etiologic and therapeutic implications. Habitual smoking was more related to dyslipidemia in middle-aged women compared with men, whereas smoking was more related to sympathetic overactivity and elevated C-reactive protein in middle-aged men compared with women. Based on the current study, blood lipid levels (especially HDL cholesterol and triglycerides) should be routinely examined for women habitual smokers for the cardiovascular risk reduction efforts. Male habitual smokers should be more aware of the acute atherothrombotic events (such as myocardial infarction, stroke) because elevated C-reactive protein suggests active inflammation predictive of an early cardiovascular outcome [43]. However, there are also reports that smoking is related to dyslipidaemia for men [44, 45] and smoking is related to elevated C-reactive protein for women as well [46]. In a study among patients with chronic coronary artery disease, cigarette smoking is associated with increased circulating proinflammatory and procoagulant markers, which are associated with endothelial dysfunction, and atherosclerosis [47, 48]. Further epidemiological studies with larger smoker samples are warranted to confirm our findings. In addition, the short follow-up period (3-year) limited our ability to some extent for detecting significant associations between risk factors and IMT progression.

Former smokers had a carotid IMT that was in-between that of never smokers and current smokers among men. On one hand, part of the adverse smoking effects is irreversible [3]. On the other hand, some atherogenic risk factors might rise among former smokers. For example, former smokers were in general more obese [49]. In our study, current smokers had lowest BMI among all three groups (especially among men), which is consistent with other reports [13]. Less general obesity in smokers may offer them some protection against progression of atherosclerosis [11]. There was also evidence that more quitters become hypertensive than non-quitters, although the groups had similar baseline blood pressure levels [50]. Weight gain subsequent to cessation probably contributed to this excess incidence of hypertension in quitters [50]. However, in the current study, we only identified the group differences in the extent of obesity but not in the levels of blood pressure.

In contrast with men, no significant difference on carotid IMT was observed for women across the three smoking status groups. However, habitual smoking was indeed associated with dyslipidemia (high triglycerides, low HDL cholesterol) in women, which is a significant atherogenic factor. How could this paradox be interpreted? Another study using the same cohort [27] revealed that cigarette smoking was related to carotid intimal thickening (which is supposed to increase IMT) and medial atrophy (which is supposed to decrease IMT) in women, which resulted in the dissociation between smoking status and overall carotid intima-medial thickness. In contrast, there was no significant smoking effect on medial thickness for men; the change in overall IMT thus reflected change in intimal thickness. Therefore, smoking is atherogenic among women as well and may even cause more harm than that among men. IMT per se may not be a valid indicator for smoking-induced atherosclerosis among women.

When both smoking status and significant mediating variables were taken into account simultaneously, smoking status (current and former smoking) was still significantly associated with the baseline IMT for men (data not shown), supporting that both direct and indirect effects were involved in smoking-induced atherosclerosis and that cigarette smoking is an independent atherogenic factor. 


\section{CONCLUSIONS}

Cigarette smoking contributed to atherosclerosis by gender-differential intermediate pathways. Some of the pathways are metabolic syndrome-associated. The effects of cigarette smoking on atherosclerosis and intermediate risk factors may be partially reversible after quitting. The findings from this study can be used to educate adult men and women to quit smoking in order to reduce overall cardiovascular risk.

\section{ACKNOWLEDGEMENTS}

This study was supported by grant \#11DT-0072 from Tobacco-Related Disease Research Program at California, USA (AZF, Principal Investigator) and grant \#HL49910 from the National Heart, Lung, and Blood Institute In USA (JHD, Principal Investigator).

\section{REFERENCES}

[1] U.S. Office on Smoking and Health. The Health Consequences of Smoking: Cardiovascular Disease; a Report of the Surgon general". Washington, D.C.: U.S. Government Printing Office 2004.

[2] Thomas GN, Chook P, Yip TW, et al. Smoking without exception adversely affects vascular structure and function in apparently healthy Chinese: Implications in global atherosclerosis prevention. Int J Cardiol 2008; 128(2): 151-3.

[3] Jiang CQ, Lao XQ, Yin P, et al. Smoking, smoking cessation and aortic arch calcification in older Chinese: the Guangzhou Biobank Cohort Study. Atherosclerosis 2009; 202(2): 529-34.

[4] Dianna JN, ed. Tobacco Smoking and Atherosclerosis: Pathogenesis and Cellular Mechanisms. New York: Plenum Press 1990.

[5] Stary HC, Chandler AB, Dinsmore RE, et al. A definition of advanced types of atherosclerotic lesions and a histological classification of atherosclerosis. A report from the Committee on Vascular Lesions of the Council on Arteriosclerosis, American Heart Association. Arterioscler Thromb Vasc Biol 1995; 15: 151231.

[6] Weitzman M, Cook S, Auinger P, et al. Tobacco smoke exposure is associated with the metabolic syndrome in adolescents. Circulation 2005; 112: 862-9.

[7] Eliasson B, Attvall S, Taskinen MR, et al. The insulin resistance syndrome in smokers is related to smoking habits. Arterioscler Thromb 1994; 14: 1946-50.

[8] Hulthe J, Bokemark L, Wikstrand J, et al. The metabolic syndrome, LDL particle size, and atherosclerosis: the Atherosclerosis and Insulin Resistance (AIR) study. Arterioscler Thromb Vasc Biol 2000; 20: 2140-7.

[9] Attvall S, Fowelin J, Lager I, et al. Smoking induces insulin resistance--a potential link with the insulin resistance syndrome. J Intern Med 1993; 233: 327-32.

[10] Henkin L, Zaccaro D, Haffner S, et al. Cigarette smoking, environmental tobacco smoke exposure and insulin sensitivity: the Insulin Resistance Atherosclerosis Study. Ann Epidemiol 1999; 9: 290-6.

[11] Hughes K, Choo M, Kuperan P, et al. Cardiovascular risk factors in relation to cigarette smoking: a population-based survey among Asians in Singapore. Atherosclerosis 1998; 137: 253-8.

[12] Rimm EB, Chan J, Stampfer MJ, et al. Prospective study of cigarette smoking, alcohol use, and the risk of diabetes in men. BMJ 1995; 310: 555-9.

[13] Wareham NJ, Ness EM, Byrne CD, et al. Cigarette smoking is not associated with hyperinsulinemia: evidence against a causal relationship between smoking and insulin resistance. Metabolism 1996; 45: 1551-6.

[14] Godsland IF, Leyva F, Walton C, et al. Associations of smoking, alcohol and physical activity with risk factors for coronary heart disease and diabetes in the first follow-up cohort of the Heart Disease and Diabetes Risk Indicators in a Screened Cohort study (HDDRISC-1). J Intern Med 1998; 244: 33-41.

[15] Howard G, Wagenknecht LE, Burke GL, et al. Cigarette smoking and progression of atherosclerosis: The Atherosclerosis Risk in Communities (ARIC) Study. JAMA 1998; 279: 119-24.
[16] Kornowski R. Impact of smoking on coronary atherosclerosis and remodeling as determined by intravascular ultrasonic imaging. Am J Cardiol 1999; 83: 443-5, A9.

[17] McGill HC, Jr., McMahan CA, Malcom GT, et al. Effects of serum lipoproteins and smoking on atherosclerosis in young men and women. The PDAY Research Group. Pathobiological Determinants of Atherosclerosis in Youth. Arterioscler Thromb Vasc Biol 1997; 17: 95-106.

[18] Gudwin AL, Padussis CJ. Smoking, age, and sex in carotid artery atherosclerosis: a review of 3,865 carotid duplex scans. Md Med J 1994; 43: 265-8

[19] Poredos P, Orehek M, Tratnik E. Smoking is associated with doserelated increase of intima-media thickness and endothelial dysfunction. Angiology 1999; 50: 201-8.

[20] Mennen LI, Balkau B, Charles MA, et al. Gender differences in the relation between fibrinogen, tissue-type plasminogen activator antigen and markers of insulin resistance: effects of smoking. D.E.S.I.R. Study Group. Data from an Epidemiological Study on Insulin Resistance Syndrome. Thromb Haemost 1999; 82: 1106-11.

[21] Visser M, Launer LJ, Deurenberg P, et al. Past and current smoking in relation to body fat distribution in older men and women. J Gerontol A Biol Sci Med Sci 1999; 54: M293-8.

[22] Primatesta P, Falaschetti E, Gupta S, et al. Association between smoking and blood pressure: evidence from the health survey for England. Hypertension 2001; 37: 187-93.

[23] Mack WJ, Selzer RH, Hodis HN, et al. One-year reduction and longitudinal analysis of carotid intima-media thickness associated with colestipol/niacin therapy. Stroke 1993; 24: 1779-83.

[24] O'Leary DH, Polak JF, Kronmal RA, et al. Carotid-artery intima and media thickness as a risk factor for myocardial infarction and stroke in older adults. Cardiovascular Health Study Collaborative Research Group. N Engl J Med 1999; 340: 14-22.

[25] Aminbakhsh A, Frohlich J, Mancini GB. Detection of early atherosclerosis with $\mathrm{B}$ mode carotid ultrasonography: assessmen of a new quantitative approach. Clin Invest Med 1999; 22: 265-74.

[26] Hodis HN, Mack WJ, LaBree L, et al. The role of carotid arterial intima-media thickness in predicting clinical coronary events. Ann Intern Med 1998; 128: 262-9.

[27] Fan AZ, Paul-Labrador M, Merz CN, et al. Smoking status and common carotid artery intima-medial thickness among middle-aged men and women based on ultrasound measurement: a cohort study. BMC Cardiovase Disord 2006; 6: 42.

[28] Fan AZ. Metabolic syndrome and progression of atherosclerosis among middle-aged US adults. J Atheroscler Thromb 2006; 13: 4654.

[29] Fan AZ, Dwyer JH. Sex differences in the relation of HDL cholesterol to progression of carotid intima-media thickness: the Los Angeles Atherosclerosis Study. Atherosclerosis 2007; 195: e191-6.

[30] Kakuyama T, Kimura S, Hashiguchi Y. Fully automated determination of HDL-cholesterol from human serum with Hitachi 911 [Abstract]. Clin Chem 1994; 40: 1104.

[31] DeLong DM, DeLong ER, Wood PD, et al. A comparison of methods for the estimation of plasma low- and very low- density lipoprotein cholesterol. The Lipid Research Clinics Prevalence Study. JAMA 1986; 256: 2372-7.

[32] Dwyer JH, Sun P, Kwong-Fu H, et al. Automated intima-media thickness: the Los Angeles Atherosclerosis Study. Ultrasound Med Biol 1998; 24: 981-7.

[33] Baron RM, Kenny DA. The moderator-mediator variable distinction in social psychological research: conceptual, strategic, and statistical considerations. J Pers Soc Psychol 1986; 51: 117382.

[34] Abe H, Yamada N. Animal models for hyperinsulinemia and insulin resistance. Ann N Y Acad Sci 2000; 902: 134-9.

[35] Kong C, Nimmo L, Elatrozy T, et al. Smoking is associated with increased hepatic lipase activity, insulin resistance, dyslipidaemia and early atherosclerosis in Type 2 diabetes. Atherosclerosis 2001; 156: $373-8$.

[36] Ostgren CJ, Lindblad U, Ranstam J, et al. Associations between smoking and beta-cell function in a non-hypertensive and nondiabetic population. Skaraborg Hypertension and Diabetes Project. Diabet Med 2000; 17: 445-50.

[37] Landsberg L. Role of the sympathetic adrenal system in the pathogenesis of the insulin resistance syndrome. Ann N Y Acad Sci 1999; 892: 84-90. 
[38] Palatini P. Heart rate as a risk factor for atherosclerosis and cardiovascular mortality: the effect of antihypertensive drugs. Drugs 1999; 57: 713-24.

[39] Pauletto P, Scannapieco G, Pessina AC. Sympathetic drive and vascular damage in hypertension and atherosclerosis. Hypertension 1991; 17: III75-81.

[40] Swedberg KB. Impact of neuroendocrine activation on coronary artery disease. Am J Cardiol 1998; 82: 8H-14H.

[41] Benowitz NL. The role of nicotine in smoking-related cardiovascular disease. Prev Med 1997; 26: 412-7.

[42] Kool MJ, Hoeks AP, Struijker Boudier HA, et al. Short- and longterm effects of smoking on arterial wall properties in habitual smokers. J Am Coll Cardiol 1993; 22: 1881-6.

[43] Whicher J, Biasucci L, Rifai N. Inflammation, the acute phase response and atherosclerosis. Clin Chem Lab Med 1999; 37: 495503.

[44] Willett W, Hennekens CH, Castelli W, et al. Effects of cigarette smoking on fasting triglyceride, total cholesterol, and HDLcholesterol in women. Am Heart J 1983; 105: 417-21.

[45] Criqui MH, Wallace RB, Heiss G, et al. Cigarette smoking and plasma high-density lipoprotein cholesterol. The Lipid Research Clinics Program Prevalence Study. Circulation 1980; 62: IV70-6.
[46] Bermudez EA, Rifai N, Buring JE, et al. Relation between markers of systemic vascular inflammation and smoking in women. Am J Cardiol 2002; 89: 1117-9.

[47] Ikonomidis I, Lekakis J, Vamvakou G, et al. Cigarette smoking is associated with increased circulating proinflammatory and procoagulant markers in patients with chronic coronary artery disease: effects of aspirin treatment. Am Heart J 2005; 149: 832-9.

[48] Lekakis JP, Ikonomidis I, Tsibida M, et al. Genetic variations of the endothelial nitric oxide synthase gene are related to increased levels of C-reactive protein and macrophage-colony stimulatingfactor in patients with coronary artery disease. Thromb Haemost 2006; 96: 520-8.

[49] Wallenfeldt K, Hulthe J, Bokemark L, et al. Carotid and femoral atherosclerosis, cardiovascular risk factors and $\mathrm{C}$-reactive protein in relation to smokeless tobacco use or smoking in 58-year-old men. J Intern Med 2001; 250: 492-501.

[50] Gerace TA, Hollis J, Ockene JK, et al. Smoking cessation and change in diastolic blood pressure, body weight, and plasma lipids. MRFIT Research Group. Prev Med 1991; 20: 602-20.

(C) Fan and Dwyer; Licensee Bentham Open.

This is an open access article licensed under the terms of the Creative Commons Attribution Non-Commercial License (http://creativecommons.org/licenses/by$\mathrm{nc} / 3.0 /$ ) which permits unrestricted, non-commercial use, distribution and reproduction in any medium, provided the work is properly cited. 